University of Massachusetts Amherst

ScholarWorks@UMass Amherst

Chemical Engineering Faculty Publication

Series

2020

\title{
Emerging concepts and tools in cell mechanomemory
}

Tanmay Lele

University of Florida

Amy Brock

University of Texas at Austin

Shelly Peyton

Follow this and additional works at: https://scholarworks.umass.edu/che_faculty_pubs

\section{Recommended Citation}

Lele, Tanmay; Brock, Amy; and Peyton, Shelly, "Emerging concepts and tools in cell mechanomemory" (2020). Annals of Biomedical Engineering. 880.

https://doi.org/10.1007/s10439-019-02412-z

This Article is brought to you for free and open access by the Chemical Engineering at ScholarWorks@UMass Amherst. It has been accepted for inclusion in Chemical Engineering Faculty Publication Series by an authorized administrator of ScholarWorks@UMass Amherst. For more information, please contact scholarworks@library.umass.edu. 


\section{Emerging concepts and tools in cell mechanomemory}

Tanmay P. Lele ${ }^{1}$, Amy Brock ${ }^{2}$, and Shelly R. Peyton ${ }^{3}$

${ }^{1}$ Department of Chemical Engineering, University of Florida, Gainesville FL

2Department of Biomedical Engineering, University of Texas at Austin, Austin TX

${ }^{3}$ Department of Chemical Engineering, University of Massachusetts, Amherst MA 


\section{Abstract}

Studying a cell's ability to sense and respond to mechanical cues has emerged as a field unto itself over the last several decades, and this research area is now populated by engineers and biologists alike. As just one example of this cell mechanosensing, fibroblasts on soft substrates have slower growth rates, smaller spread areas, lower traction forces, and slower migration speeds compared to cells on stiff substrates. This phenomenon is not unique to fibroblasts, as these behaviors, and others, on soft substrates has been shown across a variety of cell types, and reproduced in many different labs. Thus far, the field has focused on discerning the mechanisms of cell mechanosensing through ion channels, focal adhesions and integrin-binding sites to the ECM, and the cell cytoskeleton. A relatively new concept in the field is that of mechanical memory, which refers to persistent effects of mechanical stimuli long after they have been removed from said stimulus. Here, we review this literature, provide an overview of emerging substrate fabrication approaches likely to be helpful for the field, and suggest the adaption of genetic tools for studying mechanical memory. 


\section{Introduction}

The mammalian cell's response to the rigidity of the extracellular matrix (ECM) is mediated predominantly by integrins, heterodimeric receptors directly link the ECM and the cytoskeleton, and activate intracellular biochemical-signaling ${ }^{1,2}$. Synthetic, protein-, and sugar-based biopolymer material networks have been employed to study the effects of mechanics on cell behavior, including polyacrylamide ${ }^{3-5}$, Matrigel ${ }^{\mathrm{TM}} 8$, Type I Collagen ${ }^{9,10}$, poly(ethylene glycol) ${ }^{11}$, and alginate ${ }^{13}$. It has been suggested that cells respond to mechanical cues via protein structural changes ${ }^{14,15}$, alterations to complexes of many proteins (focal adhesions) ${ }^{16,17}$, or by regulating the polymerization and stabilization of several micron long cytoskeletal fibrillar polymers (actin, microtubules, and intermediate filaments) ${ }^{18-20}$.

The stiffness of the ECM can cause significant phenotypic changes in cells ${ }^{21-24}$. Structurally, cells respond to ECM stiffness via conformational changes in the focal adhesion proteins vinculin and talin, which link to the actin cytoskeleton and reveal cryptic kinase domains to initiate downstream signaling ${ }^{25,26}$. These signaling cascades from focal adhesions lead to 1) alterations in the cell cytoskeleton through Rho/ROCK and myosin-regulated tension ${ }^{27}$ that feedback to focal adhesion structures and changes in cell adhesion and motility ${ }^{28}$, and 2) changes in transcription factor activation and eventual gene expression ${ }^{29}$. The mechanisms responsible for mechanosensing include stretching of ion channels, or inside-out vs. outside-in sensing of substrate stiffness through integrins and focal adhesions ${ }^{20,30}$. These changes in cytoskeletal tension may directly control gene expression via altering force on the cell nucleus and modifying chromatin 
states ${ }^{31}$. Much is known about these short term phenotypic and longer term transcriptomic changes in cells, but less is understood about the long term changes in cell population dynamics that could be regulated by the stiffness of a cell's substrate or surroundings.

\section{What is mechano-memory?}

The effects of past mechanical cues on cells can persist long after the removal of those

cues. Such behavior has been called "mechanical memory" ${ }^{32,33}$. Early evidence of mechanical memory in the context of hydrogel stiffness came from experiments with primary rat lung fibroblasts which were cultured on a PDMS substrate of a specific stiffness for defined periods, followed by culture on PDMS substrate of a different stiffness

33. On stiff but not soft substrates, primary fibroblasts typically differentiate into a myofibroblast phenotype, characterized by expression of $\square$-smooth muscle actin and increased contractility. When these fibroblasts were cultured on stiff substrates for 3 weeks, which promoted myofibroblast differentiation, and then switched to soft substrates, the myofibroblast phenotype persisted up to the longest time point they measured (2 weeks). Conversely, culture on soft substrates for 3 weeks reduced the extent of myofibroblast differentiation when these cells were transferred to stiff substrates. These experiments showed that mechanical 'priming' or 'dosing' can induce long-term effects in cells which are irreversible on time scales of weeks after removal of the mechanical dose. 
Sustained effects of mechanical stimuli were demonstrated in the context of Yesassociated protein (YAP) and transcriptional coactivator with PDZ-binding domain (TAZ) signaling in human mesenchymal stem cells (hMSCs) ${ }^{34}$. Upon activation, YAP/TAZ localizes to the nucleus and triggers gene expression. This localization is mechanosensitive because YAP/TAZ is present in the nucleus of hMSCs on stiff substrates $(E \sim 40 \mathrm{kPa})$ but not on soft substrates $(\mathrm{E} \sim 1 \mathrm{kPa}){ }^{35}$. Culture of hMSCs on stiff tissue culture plastic (Young's modulus $\sim 3 \mathrm{GPa}$ ) caused YAP/TAZ translocation to the nucleus ${ }^{34}$. Subsequent transfer of these cells to soft substrates (Young's modulus of 2kPa) caused YAP/TAZ deactivation only when hMSCs were cultured for short times on the stiff gels ( 1 day). Longer cultures over several days on the stiff gels resulted in irreversible activation of YAP/TAZ, such that the nuclear localization of these proteins did not decrease even after culture on soft substrates for 3 days. Thus, YAP/TAZ signaling pathways are not only mechanosensitive, but their effects may persist depending on the time of 'mechanical dosing'. In addition to activation, YAP/TAZ localization caused osteogenic differentiation as measured by RUNX2 expression, again, in a mechanical dose dependent manner ${ }^{34}$. Differentiated human mammary MCF10A epithelial cells also possess the capacity for mechanical memory ${ }^{36}$. Continuous MCF10A culture on a collagen-coated polyacrylamide substrate with spatially variable stiffness showed that cells grown on the stiff portion migrated faster and retained nuclear YAP on the soft portion than cells initially on the soft substrate.

Because changes to YAP/TAZ signaling pathways in hMSCs persisted for 3 days of culture on soft substrates, it is possible that these changes are heritable across cell 
generations due to epigenetic alterations ${ }^{37}$. Anseth and coworkers investigated the effect of mechanical dosing on histone modifications. Histone acetylation in hMSCs was found to be higher on stiff substrates than on soft substrates ${ }^{38}$, and consistently, chromatin was more decondensed in these cells on stiff substrates. Furthermore, the levels of histone acetyl transferases (HATs) were higher, while those of histone deacetylases (HDACs) were lower on stiff substrates. Importantly, histone acetylation in cells cultured on stiff substrates followed by substrate softening was reversible only for short culture times (1 day) on the stiff substrates. For longer culture times (10 days), the acetylation was irreversible, such that it stayed high despite softening the gel for as long as 10 days post softening (the longest time point they measured). Collectively, these results suggest that epigenetic modifications may be a mechanism to store mechanical memory.

Alternatively, microRNA miR-21 has suggested as a key mediator of mechanical memory"39. This was demonstrated with primary rat bone marrow-derived mesenchymal stem cells cultured on PDMS substrates. Priming of MSCs on soft substrates prevented the expression of alpha smooth muscle actin when subsequently cultured on stiff substrates. Conversely, stiff-primed cells retained alpha smooth muscle actin levels when transferred to soft substrates. Interestingly, knockdown of miR-21 at the end of the stiff priming period re-sensitized cells to the soft substrates. The authors suggested that while YAP/TAZ may act as a memory storage pathway on the shorter time scales, miR-21 may provide long-term storage of mechanical memory. 
In summary, mechanical memory is the persistent effects of mechanical stimuli on cells, long after the mechanical stimulus has been removed (Figure 1). Whether the word 'memory' is appropriate for such effects is not clear, given that "memory" implies retrieval of stored information. The experiments described above certainly support the notion that mechanical stimuli can cause permanent or irreversible effects on cell differentiation, activation, and growth rates, but it stands to reason that such irreversible effects do not necessarily imply a corresponding memory pathway. We suggest that to truly prove the presence of a memory encoded in signaling pathways, the information needs to be temporarily forgotten and then remembered in the appropriate context. Studies performed so far do not appear to meet such a threshold. Perhaps the term 'persistent mechanical activation' is more appropriate for these effects.

\section{Implications for persistent mechanical activation of cancer cells}

These studies in other cell types raise the possibility that sustained exposure to changes in the ECM in vivo may impact cell functions in as yet unknown ways. This concept of a mechanical memory or a persistent mechanically activated state, though not yet addressed in the literature, has particularly important implications in cancer (Fig. 2a). During cancer initiation and progression, the tumor microenvironment stiffens via deposition and crosslinking of ECM proteins (Fig. 2b) ${ }^{40-44}$. This ECM stiffening alters the mechanical forces experienced by the resident cancer cells ${ }^{43}$. As one example, the reported moduli of breast tumors vary considerably, but can range from 100 s of $\mathrm{Pa}$ to nearly $100 \mathrm{kPa}{ }^{40,45-53}$. Further, cells that have metastasized can reside at tissue sites 
mechanically distinct from their original environments from days to months to years, likely continually adapting to this new mechanical environment over time (Fig. 2c).

This variability in stiffnesses that cancer cells can experience could have dramatic effects on cancer cell phenotypes. For example, we and others have shown that cellular response to chemotherapy and/or other targeted drugs is sensitive to the stiffness of the surrounding ECM (Fig. 2d) 7,40,48,54-62. Second, ECM stiffness plays a critical role in regulating cancer cell growth ${ }^{63-65}$ and motility ${ }^{66,67}$. However, these studies are all reports of mechanosensing in the traditional sense, where cells are cultured on tissue culture polystyrene (TCPS) and then exposed to a certain substrate stiffness for a limited experimental window. We found one study that points toward persistent mechanical activation: cancer cells were adapted to a soft biomaterial for 3 passages on polyacrylamide substrates ${ }^{12}$. They found that MDA-MB-231 cells improved their attachment and increased their cell spread area on soft substrates increasingly as they were passaged on soft substrates (Fig. 2e). These studies suggest that sustained exposure to the mechanics of the ECM can have an impact on cancer cell phenotype, but it is not yet clear if this is a phenotypic, genetic, or epigenetic response.

Common sites of breast cancer metastasis include the bone, lung, liver, and brain, which are mechanically distinct tissues ${ }^{63}$. As cancer cells disseminate, they can reside at these distant tissue sites, which have moduli far distinct from breast tissue, for decades. One example of this phenomenon is cancer dormancy. Even after apparently successful therapy, disseminated tumor cells can remain dormant for many years, often in the bone 
marrow, before outgrowth. The presence of these disseminated, quiescent tumor cells in the marrow is a marker of poor prognosis ${ }^{68,69}$. These dormant cells are also notoriously difficult to treat, and cannot be killed by the traditional chemotherapies typically given to patients with metastatic disease. Breast tumors are highly heterogeneous and drug treatment is known to enhance mutagenesis and clonal selection ${ }^{70}$. Therefore, it is quite possible that the stiffness of these distant tissue sites could be priming cancer cells for fast growth, invasion, and drug resistant qualities. This is thus far an unexplored area in need of the phenotypic, genomic, and mechanistic studies underway for fibroblasts and stem cells described earlier.

\section{Biomaterials development to investigate persistent mechanical activation of cells}

Many biomaterial systems have been developed to capture the elastic modulus of real tissue. Bioengineers, and increasing numbers of cell biologists, have used these to study how the rigidity of the microenvironment affects cell behaviors. Biomaterials made from synthetic polymer networks are attractive for this application because they have more control over mechanical properties compared to naturally-derived protein and polysaccharide biopolymers ${ }^{71}$. Polymer biomaterials such as polyacrylamide (PAA) and poly(ethylene glycol) (PEG) are excellent model systems in which to understand the biophysical aspects of cell-material relationships ${ }^{72-77}$. PAA was the first popularized material used to parse the role of substrate modulus on cell behavior ${ }^{3}$; however, its main limitation is that it cannot be used as a 3D cell culture environment. PEG, in comparison, is also not cell-degradable on its own, but can be engineered to contain hydrolytically ${ }^{78,79}$ or enzymatically degradable sites ${ }^{80}$ for $3 D$ cell culture. PEG is inherently resistant to 
protein adsorption, but can be coupled with short peptides or full-length proteins ${ }^{81,82}$ to target specific receptor-ligand interactions in cells ${ }^{73,83}$.

One innovation we have brought to the field of stiffness-tunable 2D hydrogels is based on combining PEG with the zwitterionic monomer phosphorylcholine $(P C)^{83}$. PCs and other zwitterions have been exploited for their hydrophilicity and their mimicry of cell membrane phospholipids. These features make polymers including PCs ideal for drug delivery $^{84}$, but their use in a biomaterial hydrogel has been limited ${ }^{85-87}$. PCs are extremely resistant to nonspecific protein adhesion, with performance better than polyHEMAs, acrylamides, and pyrrolidones ${ }^{88}$, and this makes them particularly attractive for the longterm culture time points required to study persistent mechanical activation of cells. Hydrogels made from combining PEG and PC can be polymerized with as little as 0.5 wt\% PEG crosslinker, resulting in a Young's modulus range over four orders of magnitude, which is also a key design criterium for studies attempting to differentiate cells based on the rigidity of the substrate.

3D hydrogels developed by us and others ${ }^{54,89,90}$ are less frequently used for long-term cell cultures. A potentially cumbersome challenge here is how, technically, to repeatedly release and re-seed cells from a 3D environment as one does during cell passaging on 2D substrata. The prime candidates for 3D hydrogels would be Matrigel, type 1 Collagen, and Fibrin. Since these hydrogels are protein-based, cells can be released by proteolytic degradation (MMPs, collagenases, trypsin, etc.). 3D synthetic hydrogel environments could be adapted for this purpose if they were to include enzymatically degradable 
crosslinks ${ }^{7,83}$. In both cases, however, enzymatic digestion of gels would be expensive for continuous passaging. Finally, a lingering challenge with any 3D gel system is the limited range of moduli these gels can achieve (typically between 10 s of $\mathrm{Pa}$ to $10 \mathrm{kPa}$ ). This is significantly lower than that achievable by 2D hydrogel systems. Additional chemistries need to be developed to achieve these higher moduli and still be appropriate for cell culture in order to study persistent mechanical activation of cells in 3D.

\section{Genetic tools to study persistent mechanical activation of cells}

\section{Epigenetic memory in transcriptomic cell states}

Gene regulatory networks determine the coordinated dynamics of gene and protein expression programs, giving rise to distinct cell states. Networks are defined by the nodes, or the molecular players including proteins or genetic elements, and the molecular interactions, or wiring diagrams, that that govern their expression and activity. A cell state is reinforced and stabilized by the feedback of these interconnected pathways. It is these self-stabilizing patterns of gene activation across the genome that account for "epigenetic memory", rendering a cell state change irreversible (or difficult to reverse), as in development and differentiation. Thus, even in the absence of the initiating stimulus that triggered a cell state change, the pattern of gene expression persists.

Transcriptomic changes may be assessed through qPCR analysis of panels of selected

genes or by RNA-seq. Importantly, advances in single cell RNA-Seq (scRNA-Seq) have 
now made possible the analysis of gene expression states in individual cells, with thousands of individual cell transcriptomes simultaneously measured. Recent studies that identify irreversible or partial irreversible gene expression changes induced by mechanical perturbations have relied on measuring only a small number of gene expression changes. As discussed earlier, one adipogenic marker (PPARg) and two osteogenic markers (alkaline phosphase and OCN) were assessed as markers of mechanical memory in mesenchymal stem cells ${ }^{34}$, and expression of actomyosin was measured in epithelial cells primed on stiff vs soft $\mathrm{ECM}^{36}$. It therefore remains to be seen, whether other dimensions of the gene regulatory network sustain heritable changes in gene expression upon removal of a mechanical signal.

\section{Measurements of epigenetic memory in chromatin}

Chromatin organization and epigenetic regulators also play key roles in the determination of cell state. To assess changes in binding of histones (such as H3K27me) and other regulatory factors, chromatin immunoprecipitation (ChIP) is performed. In this assay, proteins are covalently crosslinked to genomic DNA, providing a snapshot of histone or other protein-DNA interactions at a particular time point or in response to mechanical or biochemical signals. Following the isolation and fragmentation of chromatin, the proteinDNA complexes are isolated by binding to an antibody specific to the histone or factor of interest. The covalent crosslinks are then reversed, freeing the DNA for purification and analysis by qPCR or high-throughput sequencing (ChIP-seq). Another technique, Formaldehyde-Assisted Isolation of Regulatory Elements (FAIRE) does not rely on the availability of a specific antibody. Instead, DNA is bound to chromatin proteins by 
formaldehyde and sheared via sonication. Tightly packed chromatin regions will have abundant DNA/protein crosslinks, while DNA regions with no or few nucleosomes will have little or no crosslinked DNA/protein complexes. Quantification of this free DNA compared to a reference of total DNA sample allows the identification of the chromatin free regions. The FAIRE method can be used for the characterization of individual genomic regions or for the identification of genome-wide chromatin accessibility when coupled to deep sequencing ${ }^{91}$.

Both of these tools have been utilized to uncover epigenomic changes in response to mechanical perturbations. For example, human epidermal progenitor cells exposed to biaxial cyclic mechanical strain undergo striking changes in gene expression with nearly 4,000 genes downregulated and no genes significantly upregulated. Polycomb repressive complex (PRC) is one key player in this process, catalyzing dimethylation and trimethylation of histone 3 on Lys27 (H3K27me3) through the methyltransferase activity of Ezh1/Ezh2. Genes regulated by H3K27me3 or by the PRC pathway were overrepresented in the set of transcripts downregulated by cyclic strain ${ }^{92}$.

Another approach to quantify chromatin remodeling is the assay for transposaseaccessible chromatin sequencing (ATAC-seq), which identifies regions of open chromatin across the genome. By taking advantage of a Tn5 transposase that preferentially cleaves DNA and inserts sequencing adapters in regions of open, accessible chromatin, ATACseq enables high-throughput comparison of accessible genomic regions across samples. Subsequent next-generation sequencing and mapping of the fragments identifies putative 
regulatory regions that exhibit signatures of active transcriptional state and chromatin accessibility. In recent work, Stowers, et al. utilized this technology to compare regions of chromatin accessibility in breast cancer cells cultured in soft and stiff 3D interpenetrating networks (IPNs) of reconstituted basement membrane (rBM) matrix and alginate. This culture system enables specification of the elastic modulus independent of matrix architecture and ligand density. Differential analysis of ATAC-seq peaks revealed more than 1,600 significantly more accessible peaks for cells cultured in stiff matrices ( 2,000 Pa, corresponding to malignant tissue), with no regions found to be significantly

more accessible in soft matrices ( 100 Pa, mimicking normal mammary tissue) ${ }^{93}$. New developments in this technology now permit measurements of DNA accessibility at the single cell level by single cell ATAC-seq ${ }^{94}$. This approach could be critical for quantifying the cell-to-cell variability in epigenetic regulation of persistent mechanical activation.

\section{Tracking histories of individual cells}

To investigate the mechanisms of cellular alterations and adaptation to mechanical stimuli, it is necessary to distinguish between two broad categories of responses. Does the mechanical perturbation induce persistent, heritable changes in individual cells or is there selection (by differential survival or differential growth rates) for a subset of cells with particular pre-existing characteristics? Either of these scenarios could produce a lasting shift in the cell state of the overall population in response to a mechanical stimulus. To determine which of these general mechanisms is at work, it is critical to track individual cells in the population over time. 
While fluorescent cell labels and time-lapse microscopic imaging have enabled decades of study, these approaches are limited in the number of labels that can be monitored simultaneously and the duration over which individual cells can be observed. In 3D culture systems, tracking individual cells over days and weeks adds another layer of technical challenges. To address these limitations, novel nucleic acid-based tools have been developed to label and quantify cells and their clonal descendants within heterogeneous populations ${ }^{95}$. DNA barcoding is uniquely capable of tagging and measuring large numbers of cells over time ${ }^{96,97}$. In this approach, each cell in a population is tagged with a unique random DNA sequence that is stably integrated into the genome and thus heritable by all daughter cells. The potential space of unique sequence tags is extremely large (for example, the theoretical diversity of a library of random 20-mer barcodes is more than $10^{12}$ distinct sequences), enabling the faithful labeling of large cell populations. After stable integration (typically by viral delivery), barcode abundance can be measured by targeted next-generation sequencing of the barcode region (Fig. 3).

In recent years, this approach has been leveraged to uncover evidence of pre-existing and induced responses to various biochemical stimuli, although to our knowledge it has not yet been applied to mechanical perturbations. For example, Bhang and colleagues utilized a high-diversity DNA barcode library to investigate the response of non-small cell lung cancer cells and chronic myeloid leukemia cells to targeted growth factor pathway inhibitors ${ }^{98}$. In all samples, a population of cells resistant to therapy emerged after treatment. To determine whether this shift in the cell population phenotype was caused by selection of a pre-existing subpopulation or induction of a resistance mechanism, 
investigators measured the abundance of barcoded cells in multiple parallel replicates. NGS revealed that the very same clones consistently escaped treatment across replicates, revealing the presence of a rare pre-existing drug-resistant sub-population prior to treatment. We propose that this same approach could be used to find clones that expand in different stiffness environments as well.

Variations on DNA barcoding systems have integrated these cell labels with other molecular and genomic assays. The use of RNA-based, expressed barcode tags enables the simultaneous read-out of the barcode label alongside the captured transcriptome in single-cell RNA sequencing (scRNA-Seq) ${ }^{99-103}$. Expressed barcode systems with scRNA-Seq have been utilized to dissect the reprograming of mouse embryonic fibroblasts to induced endoderm progenitors (iEPs) ${ }^{100}$. Barcode labeling and tracking the progenitor population revealed that cells from identical lineages follow similar reprograming trajectories within a replicate, but not across replicates. These data suggest that, rather than selection of a pre-existing stable cell state, multiple cells in the starting population are able to enter a temporarily privileged cell state, in which they are primed for IEP differentiation ${ }^{100}$. Similar processes may underlie persistent mechanical activation and can now be explored in the context of heterogeneity of cell responses to substrate stiffness.

Cell barcoding platforms offer powerful new tools to dissect the histories and trajectories of individual cells and relate these to population-level shifts in gene expression and behavior. They share one limitation - they are destructive measurements due to the 
requirement of sequencing the genome to quantify barcodes. To overcome this challenge, one of us developed a functionalized variant of DNA barcoding that uses stably integrated and expressed barcoded guide-RNAs, capable of isolating live cells carrying a particular barcode label of interest ${ }^{104}$. Borrowing from synthetic biology, this approach takes advantage of a transcriptional activator variant of dCas 9 to activate a barcode-specific gene circuit and express a fluorescent reporter. This enables isolation of specific subpopulations of interest by fluorescent activated cell sorting for downstream molecular and cellular studies. This is a key technological advancement for studying persistent mechanical activation, so that we may harvest clones on soft vs. stiff environments and study important phenotypes relevant to cancer, such as their motility, growth rates, and

drug responses. This could provide a much-needed link between genotype and phenotypes in cancer related to tumor ECM stiffness.

\section{Acknowledgements}

This work was supported by an NSF CAREER to SRP (DMR1454806), a Texas 4000 Research Grant to AB and NIH R01 EB014869 to TPL. 


\section{References}

1 Hynes, R. O. Integrins: bidirectional, allosteric signaling machines. Cell 110, 673687 (2002).

2 Giancotti, F. G. \& Ruoslahti, E. Integrin signaling. Science 285, 1028-1032 (1999).

3 Pelham, R. J., Jr. \& Wang, Y. Cell locomotion and focal adhesions are regulated by substrate flexibility. Proc Natl Acad Sci U S A 94, 13661-13665 (1997).

4 Pelham, R. J., Jr. \& Wang, Y. L. Cell locomotion and focal adhesions are regulated by the mechanical properties of the substrate. Biol Bull 194, 348-349; discussion 349-350 (1998).

5 Engler, A. et al. Substrate compliance versus ligand density in cell on gel responses. Biophys J 86, 617-628 (2004).

6 Provenzano, P. P. et al. Collagen density promotes mammary tumor initiation and progression. BMC Med 6, 11, doi:10.1186/1741-7015-6-11 (2008).

7 Nguyen, T. V., Sleiman, M., Moriarty, T., Herrick, W. G. \& Peyton, S. R. Sorafenib resistance and JNK signaling in carcinoma during extracellular matrix stiffening. Biomaterials 35, 5749-5759, doi:10.1016/j.biomaterials.2014.03.058 (2014).

8 Deroanne, C. F., Lapiere, C. M. \& Nusgens, B. V. In vitro tubulogenesis of endothelial cells by relaxation of the coupling extracellular matrix-cytoskeleton. Cardiovasc Res 49, 647-658 (2001).

9 Wozniak, M. A., Desai, R., Solski, P. A., Der, C. J. \& Keely, P. J. ROCK-generated contractility regulates breast epithelial cell differentiation in response to the physical properties of a three-dimensional collagen matrix. J Cell Biol 163, 583595 (2003). 
10 Halliday, N. L. \& Tomasek, J. J. Mechanical properties of the extracellular matrix influence fibronectin fibril assembly in vitro. Exp Cell Res 217, 109-117 (1995).

11 Peyton, S. R., Kim, P. D., Ghajar, C. M., Seliktar, D. \& Putnam, A. J. The effects of matrix stiffness and RhoA on the phenotypic plasticity of smooth muscle cells in a 3-D biosynthetic hydrogel system. Biomaterials 29, 2597-2607, doi:10.1016/j.biomaterials.2008.02.005 (2008).

12 Syed, S., Schober, J., Blanco, A. \& Zustiak, S. P. Morphological adaptations in breast cancer cells as a function of prolonged passaging on compliant substrates. PLoS One 12, e0187853, doi:10.1371/journal.pone.0187853 (2017).

13 Rowley, J. A., Madlambayan, G. \& Mooney, D. J. Alginate hydrogels as synthetic extracellular matrix materials. Biomaterials 20, 45-53 (1999).

14 Mierke, C. T. et al. Mechano-coupling and regulation of contractility by the vinculin tail domain. Biophys J 94, 661-670, doi:biophysj.107.108472 (2008).

15 del Rio, A. et al. Stretching single talin rod molecules activates vinculin binding. Science 323, 638-641, doi:323/5914/63810.1126/science.1162912 (2009).

16 Balaban, N. Q. et al. Force and focal adhesion assembly: a close relationship studied using elastic micropatterned substrates. Nat Cell Bio/ 3, 466-472 (2001).

17 Riveline, D. et al. Focal contacts as mechanosensors: externally applied local mechanical force induces growth of focal contacts by an mDia1-dependent and ROCK-independent mechanism. J Cell Biol 153, 1175-1186 (2001).

18 Stamenovic, D. \& Ingber, D. E. Models of cytoskeletal mechanics of adherent cells. Biomech Model Mechanobiol 1, 95-108 (2002). 
19 Brangwynne, C. P. et al. Microtubules can bear enhanced compressive loads in living cells because of lateral reinforcement. J Cell Biol 173, 733-741 (2006).

20 Kumar, S. et al. Viscoelastic retraction of single living stress fibers and its impact on cell shape, cytoskeletal organization, and extracellular matrix mechanics. Biophys J 90, 3762-3773 (2006).

21 Discher, D. E., Janmey, P. \& Wang, Y.-I. Tissue Cells Feel and Respond to the Stiffness of Their Substrate. Science 310, 1139-1143 (2005).

22 Chaudhuri, O. et al. Hydrogels with tunable stress relaxation regulate stem cell fate and activity. Nat Mater 15, 326-334, doi:10.1038/nmat4489 (2016).

23 Hwang, J. H. et al. Extracellular Matrix Stiffness Regulates Osteogenic Differentiation through MAPK Activation. PLoS One 10, e0135519, doi:10.1371/journal.pone.0135519 (2015).

24 Kolahi, K. S. \& Mofrad, M. R. Mechanotransduction: a major regulator of homeostasis and development. Wiley Interdiscip Rev Syst Biol Med 2, 625-639, doi:10.1002/wsbm.79 (2010).

25 Yao, M. et al. The mechanical response of talin. Nat Commun 7, 11966, doi:10.1038/ncomms11966 (2016).

26 Holle, A. W. et al. In situ mechanotransduction via vinculin regulates stem cell differentiation. Stem Cells 31, 2467-2477, doi:10.1002/stem.1490 (2013).

27 Wu, J., Dickinson, R. B. \& Lele, T. P. Investigation of in vivo microtubule and stress fiber mechanics with laser ablation. Integr Biol (Camb) 4, 471-479, doi:10.1039/c2ib20015e (2012). 
28 Peyton, S. R. \& Putnam, A. J. Extracellular matrix rigidity governs smooth muscle cell motility in a biphasic fashion. Journal of Cellular Physiology 204, 198-209, doi:10.1002/jcp.20274 (2005).

29 Schwartz, A. D., Hall, C. L., Barney, L. E., Babbitt, C. C. \& Peyton, S. R. Integrin alpha6 and EGFR signaling converge at mechanosensitive calpain 2. Biomaterials 178, 73-82, doi:10.1016/j.biomaterials.2018.05.056 (2018).

30 Matthews, B. D., Overby, D. R., Mannix, R. \& Ingber, D. E. Cellular adaptation to mechanical stress: role of integrins, Rho, cytoskeletal tension and mechanosensitive ion channels. J Cell Sci 119, 508-518 (2006).

31 Tajik, A. et al. Transcription upregulation via force-induced direct stretching of chromatin. Nat Mater 15, 1287-1296, doi:10.1038/nmat4729 (2016).

32 Chan, W. L., Silberstein, J. \& Hai, C. M. Mechanical strain memory in airway smooth muscle. Am J Physiol Cell Physiol 278, C895-904, doi:10.1152/ajpcell.2000.278.5.C895 (2000).

33 Balestrini, J. L., Chaudhry, S., Sarrazy, V., Koehler, A. \& Hinz, B. The mechanical memory of lung myofibroblasts. Integr Biol (Camb) 4, 410-421, doi:10.1039/c2ib00149g (2012).

34 Yang, C., Tibbitt, M. W., Basta, L. \& Anseth, K. S. Mechanical memory and dosing influence stem cell fate. Nat Mater 13, 645-652, doi:10.1038/nmat3889 (2014).

35 Dupont, S. et al. Role of YAP/TAZ in mechanotransduction. Nature 474, 179-183, doi:10.1038/nature10137 (2011). 
36 Nasrollahi, S. et al. Past matrix stiffness primes epithelial cells and regulates their future collective migration through a mechanical memory. Biomaterials 146, 146155, doi:10.1016/j.biomaterials.2017.09.012 (2017).

37 Zenk, F. et al. Germ line-inherited H3K27me3 restricts enhancer function during maternal-to-zygotic transition. Science 357, 212-216, doi:10.1126/science.aam5339 (2017).

38 Killaars, A. R. et al. Extended Exposure to Stiff Microenvironments Leads to Persistent Chromatin Remodeling in Human Mesenchymal Stem Cells. Adv Sci (Weinh) 6, 1801483, doi:10.1002/advs.201801483 (2019).

$39 \mathrm{Li}, \mathrm{C} . \mathrm{X}$. et al. MicroRNA-21 preserves the fibrotic mechanical memory of mesenchymal stem cells. Nat Mater 16, 379-389, doi:10.1038/nmat4780 (2017).

40 Levental, K. R. et al. Matrix crosslinking forces tumor progression by enhancing integrin signaling. Cell 139, 891-906, doi:10.1016/j.cell.2009.10.027 (2009).

41 Cox, T. R. \& Erler, J. T. Remodeling and homeostasis of the extracellular matrix: implications for fibrotic diseases and cancer. Dis Model Mech 4, 165-178, doi:10.1242/dmm.004077 (2011).

42 Acerbi, I. et al. Human breast cancer invasion and aggression correlates with ECM stiffening and immune cell infiltration. Integr Biol (Camb) 7, 1120-1134, doi:10.1039/c5ib00040h (2015).

43 Katira, P., Bonnecaze, R. T. \& Zaman, M. H. Modeling the mechanics of cancer: effect of changes in cellular and extra-cellular mechanical properties. Front Oncol 3, 145, doi:10.3389/fonc.2013.00145 (2013). 
44 Seo, B. R. et al. Obesity-dependent changes in interstitial ECM mechanics promote breast tumorigenesis. Sci Trans Med 7 (2015).

45 Huwart, L. et al. MR elastography of liver fibrosis: preliminary results comparing spin-echo and echo-planar imaging. Eur Radiol 18, 2535-2541, doi:10.1007/s00330-008-1051-5 (2008).

46 Huwart, L. et al. Liver fibrosis: non-invasive assessment with MR elastography. NMR Biomed 19, 173-179, doi:10.1002/nbm.1030 (2006).

47 Tilghman, R. W. et al. Matrix rigidity regulates cancer cell growth and cellular phenotype. PLoS One 5, e12905, doi:10.1371/journal.pone.0012905 (2010).

48 Paszek, M. J. et al. Tensional homeostasis and the malignant phenotype. Cancer Cell 8, 241-254 (2005).

49 Paszek, M. J. \& Weaver, V. M. The tension mounts: mechanics meets morphogenesis and malignancy. J Mammary Gland Biol Neoplasia 9, 325-342 (2004).

50 Bretscher, M. S. On the shape of migrating cells--a 'front-to-back' model. J Cell Sci 121, 2625-2628, doi:10.1242/jcs.031120 (2008).

51 Iwanicki, M. P. et al. Ovarian cancer spheroids use myosin-generated force to clear the mesothelium. Cancer Discov 1, 144-157 (2011).

52 Provenzano, P. P. et al. Collagen reorganization at the tumor-stromal interface facilitates local invasion. BMC Med 4, 38, doi:10.1186/1741-7015-4-38 (2006).

53 Kostic, A., Lynch, C. D. \& Sheetz, M. P. Differential matrix rigidity response in breast cancer cell lines correlates with the tissue tropism. PLoS One 4, e6361, doi:10.1371/journal.pone.0006361 (2009). 
54 Schwartz, A. D. et al. A biomaterial screening approach reveals microenvironmental mechanisms of drug resistance. Integr Biol (Camb) 9, 912924, doi:10.1039/c7ib00128b (2017).

55 Tokuda, E. Y., Jones, C. E. \& Anseth, K. S. PEG-peptide hydrogels reveal differential effects of matrix microenvironmental cues on melanoma drug sensitivity. Integr Biol (Camb) 9, 76-87, doi:10.1039/c6ib00229c (2017).

56 Zustiak, S., Nossal, R. \& Sackett, D. L. Multiwell stiffness assay for the study of cell responsiveness to cytotoxic drugss. Biotechnol Bioeng 9999, 1-8, doi:10.1002/bit.25097 (2013).

57 Chang, C. C. et al. Regulation of metastatic ability and drug resistance in pulmonary adenocarcinoma by matrix rigidity via activating c-Met and EGFR. Biomaterials 60, 141-150, doi:10.1016/j.biomaterials.2015.04.058 (2015).

58 Lam, C. R. et al. A 3D biomimetic model of tissue stiffness interface for cancer drug testing. Mol Pharm 11, 2016-2021, doi:10.1021/mp500059q (2014).

59 Hirata, E. et al. Intravital imaging reveals how BRAF inhibition generates drugtolerant microenvironments with high integrin beta1/FAK signaling. Cancer Cell 27, 574-588, doi:10.1016/j.ccell.2015.03.008 (2015).

60 Zustiak, S. P. et al. Three-dimensional matrix stiffness and adhesive ligands affect cancer cell response to toxins. Biotechnol Bioeng 113, 443-452, doi:10.1002/bit.25709 (2016).

61 Mih, J. D. et al. A multiwell platform for studying stiffness-dependent cell biology. PLoS One 6, e19929, doi:10.1371/journal.pone.0019929 (2011). 
62 McGrail DJ, K. Q., Dawson MR. Metastatic ovarian cancer cell malignancy is increased on soft matrices through a mechanosensitive Rho/ROCK pathway. $J$ Cell Sci 127, 2621-2626 (2014).

63 Duda, D. G. et al. Malignant cells facilitate lung metastasis by bringing their own soil. P Natl Acad Sci USA 107, 21677-21682, doi:10.1073/pnas.1016234107 (2010).

64 Samuel, M. S. et al. Actomyosin-mediated cellular tension drives increased tissue stiffness and beta-catenin activation to induce epidermal hyperplasia and tumor growth. Cancer Cell 19, 776-791, doi:10.1016/j.ccr.2011.05.008 (2011).

65 Shen, Y. I. et al. Hyaluronic acid hydrogel stiffness and oxygen tension affect cancer cell fate and endothelial sprouting. Biomaterials science 2, 655-665, doi:10.1039/C3BM60274E (2014).

66 Swaminathan, V. et al. Mechanical stiffness grades metastatic potential in patient tumor cells and in cancer cell lines. Cancer Res 71, 5075-5080, doi:10.1158/00085472.CAN-11-0247 (2011).

67 Fenner, J. et al. Macroscopic stiffness of breast tumors predicts metastasis. Sci Rep 4, 5512, doi:10.1038/srep05512 (2014).

68 Kim, B. S., Putnam, A. J., Kulik, T. J. \& Mooney, D. J. Optimizing seeding and culture methods to engineer smooth muscle tissue on biodegradable polymer matrices. Biotechnol Bioeng 57, 46-54 (1998).

69 Bokoch, G. M., Bohl, B. P. \& Chuang, T. H. Guanine nucleotide exchange regulates membrane translocation of Rac/Rho GTP-binding proteins. J Biol Chem 269, 31674-31679 (1994). 
70 Brady, S. W. et al. Combating subclonal evolution of resistant cancer phenotypes. Nat Commun 8, 1231, doi:10.1038/s41467-017-01174-3 (2017).

71 Kim, H. D. \& Peyton, S. R. Bio-inspired materials for parsing matrix physicochemical control of cell migration: a review. Integr Biol (Camb) 4, 37-52, doi:10.1039/c1ib00069a (2012).

72 Peyton, S. R., Kim, P. D., Ghajar, C. M., Seliktar, D. \& Putnam, A. J. The effects of matrix stiffness and RhoA on the phenotypic plasticity of smooth muscle cells in a 3-D biosynthetic hydrogel system. Biomaterials 29, 2597-2607, doi:10.1016/j.biomaterials.2008.02.005 (2008).

73 Peyton, S. R., Raub, C. B., Keschrumrus, V. P. \& Putnam, A. J. The use of poly(ethylene glycol) hydrogels to investigate the impact of ECM chemistry and mechanics on smooth muscle cells. Biomaterials 27, 4881-4893, doi:10.1016/j.biomaterials.2006.05.012 (2006).

74 Khatiwala, C. B., Kim, P. D., Peyton, S. R. \& Putnam, A. J. ECM compliance regulates osteogenesis by influencing MAPK signaling downstream of RhoA and ROCK. J Bone Miner Res 24, 886-898, doi:10.1359/jbmr.081240 (2009).

75 Khatiwala, C. B., Peyton, S. R., Metzke, M. \& Putnam, A. J. The regulation of osteogenesis by ECM rigidity in MC3T3-E1 cells requires MAPK activation. $J$ Cell Physiol 211, 661-672, doi:10.1002/jcp.20974 (2007).

76 Peyton, S. R. et al. Marrow-derived stem cell motility in 3D synthetic scaffold is governed by geometry along with adhesivity and stiffness. Biotechnology and bioengineering 108, 1181-1193, doi:10.1002/bit.23027 (2011). 
77 Peyton, S. R. \& Putnam, A. J. Extracellular matrix rigidity governs smooth muscle cell motility in a biphasic fashion. J Cell Physiol 204, 198-209, doi:10.1002/jcp.20274 (2005).

78 Metters, A. T., Anseth, K. S. \& Bowman, C. N. Fundamental studies of a novel, biodegradable PEG-b-PLA hydrogel. Polymer 41, 3993-4004 (2000).

79 Zustiak, S. P. \& Leach, J. B. Hydrolytically degradable poly(ethylene glycol) hydrogel scaffolds with tunable degradation and mechanical properties. Biomacromolecules 11, 1348-1357, doi:10.1021/bm100137q (2010).

80 Raeber, G. P., Lutolf, M. P. \& Hubbell, J. A. Molecularly engineered PEG hydrogels: a novel model system for proteolytically mediated cell migration. Biophys J 89, 1374-1388 (2005).

81 Wylie, R. G. et al. Spatially controlled simultaneous patterning of multiple growth factors in three-dimensional hydrogels. Nat Mater 10, 799-806, doi:10.1038/nmat3101 (2011).

82 Masters, K. S. Covalent growth factor immobilization strategies for tissue repair and regeneration. Macromol Biosci 11, 1149-1163, doi:10.1002/mabi.201000505 (2011).

83 Herrick, W. G. et al. PEG-phosphorylcholine hydrogels as tunable and versatile platforms for mechanobiology. Biomacromolecules 14, 2294-2304, doi:10.1021/bm400418g (2013).

84 Iwasaki, Y. \& Ishihara, K. Phosphorylcholine-containing polymers for biomedical applications. Anal Bioanal Chem 381, 534-546, doi:Doi 10.1007/S00216-0042805-9 (2005). 
$85 \mathrm{Xu}, \mathrm{Y}$. et al. The biological performance of cell-containing phospholipid polymer hydrogels in bulk and microscale form. Biomaterials 31, 8839-8846, doi:10.1016/j.biomaterials.2010.07.106 (2010).

86 Kiritoshi, Y. \& Ishihara, K. Preparation of cross-linked biocompatible poly(2methacryloyloxyethyl phosphorylcholine) gel and its strange swelling behavior in water/ethanol mixture. J Biomater Sci Polym Ed 13, 213-224 (2002).

87 Shimizu, T., Goda, T., Minoura, N., Takai, M. \& Ishihara, K. Super-hydrophilic silicone hydrogels with interpenetrating poly(2-methacryloyloxyethyl phosphorylcholine) networks. Biomaterials 31, 3274-3280, doi:10.1016/j.biomaterials.2010.01.026 (2010).

88 Ishihara, K. et al. Why do phospholipid polymers reduce protein adsorption? Journal of Biomedical Materials Research 39, 323-330 (1998).

89 Gencoglu, M. F. et al. Comparative Study of Multicellular Tumor Spheroid Formation Methods and Implications for Drug Screening. ACS Biomater Sci Eng 4, 410-420, doi:10.1021/acsbiomaterials.7b00069 (2018).

90 Jansen, L., McCarthy, T., Lee, M. \& Peyton, S. A synthetic, three-dimensional bone marrow hydrogel. bioRxiv, doi:https://doi.org/10.1101/275842 (2018).

91 Giresi, P. G., Kim, J., McDaniell, R. M., lyer, V. R. \& Lieb, J. D. FAIRE (Formaldehyde-Assisted Isolation of Regulatory Elements) isolates active regulatory elements from human chromatin. Genome Res 17, 877-885, doi:10.1101/gr.5533506 (2007). 
92 Le, H. Q. et al. Mechanical regulation of transcription controls Polycomb-mediated gene silencing during lineage commitment. Nat Cell Biol 18, 864-875, doi:10.1038/ncb3387 (2016).

93 Stowers, R. S. et al. Matrix stiffness induces a tumorigenic phenotype in mammary epithelium through changes in chromatin accessibility. Nat Biomed Eng, doi:10.1038/s41551-019-0420-5 (2019).

94 Buenrostro, J. D. et al. Single-cell chromatin accessibility reveals principles of regulatory variation. Nature 523, 486-490, doi:10.1038/nature14590 (2015).

95 Woodworth, M. B., Girskis, K. M. \& Walsh, C. A. Building a lineage from single cells: genetic techniques for cell lineage tracking. Nat Rev Genet 18, 230-244, doi:10.1038/nrg.2016.159 (2017).

96 Kebschull, J. M. \& Zador, A. M. Cellular barcoding: lineage tracing, screening and beyond. Nat Methods 15, 871-879, doi:10.1038/s41592-018-0185-x (2018).

97 Blundell, J. R. \& Levy, S. F. Beyond genome sequencing: lineage tracking with barcodes to study the dynamics of evolution, infection, and cancer. Genomics 104, 417-430, doi:10.1016/j.ygeno.2014.09.005 (2014).

98 Bhang, H. E. et al. Studying clonal dynamics in response to cancer therapy using high-complexity barcoding. Nat Med 21, 440-448, doi:10.1038/nm.3841 (2015).

99 Alemany, A., Florescu, M., Baron, C. S., Peterson-Maduro, J. \& van Oudenaarden, A. Whole-organism clone tracing using single-cell sequencing. Nature 556, 108112, doi:10.1038/nature25969 (2018).

100 Biddy, B. A. et al. Single-cell mapping of lineage and identity in direct reprogramming. Nature 564, 219-224, doi:10.1038/s41586-018-0744-4 (2018). 
101 Raj, B., Gagnon, J. A. \& Schier, A. F. Large-scale reconstruction of cell lineages using single-cell readout of transcriptomes and CRISPR-Cas9 barcodes by scGESTALT. Nature protocols 13, 2685-2713, doi:10.1038/s41596-018-0058-x (2018).

102 Raj, B. et al. Simultaneous single-cell profiling of lineages and cell types in the vertebrate brain. Nat Biotechno/ 36, 442-450, doi:10.1038/nbt.4103 (2018).

103 Rodriguez-Fraticelli, A. E. et al. Clonal analysis of lineage fate in native haematopoiesis. Nature 553, 212-216, doi:10.1038/nature25168 (2018).

104 Al'Khafaji, A. M., Deatherage, D. \& Brock, A. Control of Lineage-Specific Gene Expression by Functionalized gRNA Barcodes. ACS Synth Biol 7, 2468-2474, doi:10.1021/acssynbio.8b00105 (2018). 


\section{Figure Legends}

Figure 1. Schematic of a possible mechano-memory experiment. a) Traditional mechanosensing experiments involve cell lines from standard culture on plastic, or primary cells plated on a substrate with some stiffness (ii) for a short period of time. Their ability to mechanosense is determined by different phenotypic responses to different stiffnesses. b) More recently, cells have been cultured on gels of defined stiffnesses for much longer periods of times (days, weeks, or even months) on a substrate of stiffness ii to drive longer phenotypic changes. c) To determine "mechano-memory" cells are cultured on a substrate of stiffness ii and switched to a substrate of stiffness iii. If the cells are mechanosensitive, then the phenotype as well as biochemical pathways will be different between $\mathrm{i}$, ii, and iii. If the phenotype measured in ii persists on iii, then the experiment leads to the conclusion of persistent mechanical effects long after removal of the ii stimulus. This has been termed "mechanical memory".

Figure 2. Stiffness and mechano-memory in cancer. a) During metastasis, cancer cells see a variety of different stiffness environments that could impact their phenotype. Further, their residence time at these different locations will vary. b) The stiffness of the primary tumor site is known to increase as the tumor grows, due to increased density of fibrillar collagens (Tumor-associated collagen signatures, TACS). Figure reproduced with permission from ${ }^{6} . \mathrm{c}$ ) In breast cancer, the stiffness of the tissues to which cells most commonly metastasize ranges several orders of magnitude. d) The stiffness of the culture substrate (x-axis) impacts cancer cell (colored lines) response to therapy (y-axis) in a cellline dependent manner. Figure adapted with permission from ${ }^{7}$. e) The Zustiak lab has 
reported that passaging MDA-MB-231 cells continually on soft substrates causes adaptation to those substrates. Figure reproduced with permission from ${ }^{12}$.

Fig 3. Cell barcoding approach to quantify effects of mechanics on cell populations. A population of cells is labeled with DNA barcodes and expanded. The abundance of each label can be measured by targeted sequencing of the barcode region of the genome (left). If the barcode is also an expressed sequence (right), it may be captured in workflows that analyze transcripts, such as by bead capture in single cell RNA-seq. 